\title{
Convexity and smoothness of scale functions and de Finetti's control problem
}

\author{
Andreas E. Kyprianou, Víctor Rivero ${ }^{\dagger}$ and Renming Song ${ }^{\ddagger}$
}

September 4, 2021

\begin{abstract}
Under appropriate conditions, we obtain smoothness and convexity properties of $q$-scale functions for spectrally negative Lévy processes. Our method appeals directly to very recent developments in the theory of potential analysis of subordinators. As an application of the latter results to scale functions, we are able to continue the very recent work of [2] and [26]. We strengthen their collective conclusions by showing, amongst other results, that whenever the Lévy measure has a density which is log convex then for $q>0$ the scale function $W^{(q)}$ is convex on some half line $\left(a^{*}, \infty\right)$ where $a^{*}$ is the largest value at which $W^{(q) \prime}$ attains its global minimum. As a consequence we deduce that de Finetti's classical actuarial control problem is solved by a barrier strategy where the barrier is positioned at height $a^{*}$.
\end{abstract}

AMS 2000 Mathematics Subject Classification: Primary 60J99; secondary 93E20, $60 \mathrm{G} 51$.

Keywords and phrases: Potential analysis special Bernstein function, scale functions for spectrally negative Lévy processes, control theory.

\section{Introduction}

Recently there has been considerable progress in the potential analysis of subordinators, in particular with the identification of a natural class of subordinators known as special subordinators (see for example [30, 32]). At the same time, there has been a growing body

\footnotetext{
*Department of Mathematical Sciences, University of Bath, Claverton Down, Bath, BA2 7AY, U.K. E-mail: a.kyprianou@bath.ac.uk

${ }^{\dagger}$ Department of Mathematical Sciences, University of Bath, Claverton Down, Bath, BA2 7AY, U.K. and Centro de Investigación en Matemáticas (CIMAT A.C.), Calle Jalisco s/n, Col. Valenciana, A. P. 402, C.P. 36000, Guanajuato, Gto. MEXICO. E-mail: riverovm@gmail.com

† Department of Mathematics,University of Illinois, 1409 W. Green Street, Urbana, IL 61801, USA . E-mail: rsong@math.uiuc.edu
} 
of literature concerning actuarial mathematics which explores the interaction of classical models of risk and fine properties of Lévy processes with a view to gaining new results on both sides (see for example [2, 10, 18, 19, 23, 22, 24, 26, 28, 31]).

In this paper we shall marry some of these developments together. We will use new potential analytic considerations found in, for example [30, 32, to understand better smoothness properties of scale functions for spectrally negative Lévy processes. This builds on other recent developments which closely link the theory of scale functions to potential analysis of subordinators, see [17] and [25]. In turn this will allow us to solve de Finetti's classical actuarial control problem for a much larger class of driving spectrally negative Lévy processes than previously known. For the remainder of this introduction we shall elaborate on the latter in more detail before moving on to our results and their proofs.

Henceforth we assume that $X=\left(X_{t}: t \geq 0\right)$ is a spectrally negative Lévy process with Lévy triplet given by $(\gamma, \sigma, \Pi)$, where $\gamma \in \mathbb{R}, \sigma \geq 0$ and $\Pi$ is a measure on $(0, \infty)$ satisfying

$$
\int_{0}^{\infty}\left(1 \wedge x^{2}\right) \Pi(d x)<\infty
$$

The Laplace exponent of $X$ is given by

$$
\psi(\theta)=\log \left(\mathbb{E}\left(e^{\theta X_{1}}\right)\right)=\gamma \theta+\frac{1}{2} \sigma^{2} \theta^{2}-\int_{0}^{\infty}\left(1-e^{-\theta x}-\theta x 1_{\{0<x<1\}}\right) \Pi(d x) .
$$

(The reader will note that, for convenience, we have arranged the representation of the Laplace exponent in such a way that the support of the Lévy measure is positive even though the process experiences only negative jumps). Let $\Phi(0)$ be the largest real root of $\psi$ and recall that $\Phi(0)>0$ if and only if $X$ drifts to $-\infty$, or equivalently $\psi^{\prime}(0+)<0$. The restriction $\psi:[\Phi(0), \infty) \rightarrow[0, \infty)$ is a bijection whose inverse will be denoted by $\Phi$.

Let $\phi$ be the Laplace exponent of the descending ladder height subordinator $\widehat{H}=\left(\widehat{H}_{s}, s \geq\right.$ 0 ) associated to $X$. Standard theory dictates that $\phi$ and $\psi$ are related by the Wiener-Hopf factorization

$$
\psi(\theta)=(\theta-\Phi(0)) \phi(\theta), \quad \theta \geq 0,
$$

where $\phi$ satisfies

$$
\phi(\theta)=\kappa+\mathrm{d} \theta+\int_{0}^{\infty}\left(1-e^{-\theta x}\right) \Upsilon(x) d x, \quad \theta \geq 0,
$$

with $\mathrm{d}=\sigma^{2} / 2, \kappa \geq 0, \kappa \Phi(0)=0$ and $\Upsilon:(0, \infty) \rightarrow(0, \infty)$ a function such that $\int_{0}^{\infty}(1 \wedge$ $x) \Upsilon(x) d x<\infty$. Moreover,

$$
\bar{\Pi}(x):=\int_{x}^{\infty} \Pi(d x) \text { and } \bar{\Upsilon}(x):=\int_{x}^{\infty} \Upsilon(z) d z=e^{\Phi(0) x} \int_{x}^{\infty} e^{-\Phi(0) z} \bar{\Pi}(z) d z, \quad x>0
$$


where the last equality is also a well established fact. The Wiener-Hopf factorization for $\psi$, in its Laplace transform form also states that $\psi, \Phi$ and the Laplace exponent of the bivariate descending ladder processes, say $\widehat{\kappa}: \mathbb{R}^{+} \times \mathbb{R}^{+} \mapsto \mathbb{R}$, are related by the equation

$$
\widehat{\kappa}(\alpha, \beta)=c \frac{\alpha-\psi(\beta)}{\Phi(\alpha)-\beta}, \quad \alpha, \beta \geq 0,
$$

where $c>0$ is an arbitrary constant depending on the normalization of local time at the infiumum. Without loss of generality we can and will suppose that it is equal to 1.

A key object in the fluctuation theory of spectrally negative Lévy processes and its applications is the scale functions. For each $q \geq 0$ the so called $q$-scale function of $X$, $W^{(q)}: \mathbb{R} \rightarrow[0, \infty)$, is the unique function such that $W^{(q)}(x)=0$ for $x<0$ and on $[0, \infty)$ is a strictly increasing and continuous function whose Laplace transform is given by

$$
\int_{0}^{\infty} e^{-\theta x} W^{(q)}(x) d x=\frac{1}{\psi(\theta)-q}, \quad \theta>\Phi(q) .
$$

In the last 10 years or so the use of scale functions has proved to be of great importance in a wide variety of applied probability models driven by spectrally negative Lévy processes. We refer to [20], [17] and [25] for a recent overview of their presence in the literature. As alluded to above, we are concerned here in particular with their importance in one of the most classical problems of modern actuarial mathematics: de Finetti's control problem.

Recall that the classical Cramér-Lundberg risk process corresponds to a spectrally negative Lévy process $X$ taking the form of a compound Poisson process with arrival rate $\lambda>0$ and negative jumps, corresponding to claims, having common distribution function $F$ with finite mean $1 / \mu$ as well as a drift $c>0$, corresponding to a steady income due to premiums. It is usual to assume the net profit condition $c-\lambda / \mu>0$ which says nothing other than $\psi^{\prime}(0+)>0$.

An offshoot of the classical ruin problem for the Cramér-Lundberg process was introduced by de Finetti [7]. His intention was to make the study of ruin under the Cramér-Lundberg dynamics more realistic by introducing the possibility that dividends are paid out to share holders up to the moment of ruin. Further, the payment of dividends should be made in such a way as to optimize the expected net present value of the total dividends paid to the shareholders from time zero until ruin. Mathematically speaking, de Finetti's dividend problem amounts to solving a control problem which we state in the next paragraph but within the framework of the general Lévy insurance risk process. The latter process is nothing more than a general spectrally negative Lévy process which respects the analogue of the net profit condition, namely $\psi^{\prime}(0+)>0$ (although the latter is not necessary in what follows).

Suppose that $X$ is a general spectrally negative Lévy process (no assumption is made on its long term behaviour) with probabilities $\left\{\mathbb{P}_{x}: x \in \mathbb{R}\right\}$ such that under $\mathbb{P}_{x}$ we have 
$X_{0}=x$ with probability one. (For convenience we shall write $\mathbb{P}_{0}=\mathbb{P}$ ). Let $\xi=\left\{L_{t}^{\xi}: t \geq 0\right\}$ be a dividend strategy consisting of a left-continuous non-negative non-decreasing process adapted to the (completed and right continuous) filtration $\left\{\mathcal{F}_{t}: t \geq 0\right\}$ of $X$. The quantity $L_{t}^{\xi}$ thus represents the cumulative dividends paid out up to time $t$ by the insurance company whose risk process is modelled by $X$. The controlled risk process when taking into account of the dividend strategy $\xi$ is thus $U^{\xi}=\left\{U_{t}^{\xi}: t \geq 0\right\}$ where $U_{t}^{\xi}=X_{t}-L_{t}^{\xi}$. Write $\sigma^{\xi}=$ $\inf \left\{t>0: U_{t}^{\xi}<0\right\}$ for the time at which ruin occurs when the dividend payments are taking into account. A dividend strategy is called admissible if at any time before ruin a lump sum dividend payment is smaller than the size of the available reserves; in other words $L_{t+}^{\xi}-L_{t}^{\xi} \leq \max \left\{U_{t}^{\xi}, 0\right\}$ for $t \leq \sigma^{\xi}$. Denoting the set of all admissible strategies by $\Xi$, the expected value discounted at rate $q>0$ of the dividend policy $\xi \in \Xi$ with initial capital $x \geq 0$ is given by

$$
v_{\xi}(x)=\mathbb{E}_{x}\left(\int_{\left[0, \sigma^{\xi}\right]} e^{-q t} d L_{t}^{\xi}\right),
$$

where $\mathbb{E}_{x}$ denotes expectation with respect to $\mathbb{P}_{x}$ and $q>0$ is a fixed rate. De Finetti's dividend problem consists of solving the following stochastic control problem: characterize

$$
v^{*}(x):=\sup _{\xi \in \Xi} v_{\xi}(x)
$$

and, further, if it exists, establish a strategy $\xi^{*}$ such that $v^{*}(x)=v_{\xi^{*}}(x)$.

This problem was considered by Gerber [13] who proved that, for the Cramér-Lundberg model with exponentially distributed jumps, the optimal value function is a result of a barrier strategy. That is to say, a strategy of the form $L_{t}^{a}=a \vee \bar{X}_{t}-a$ for some $a \geq 0$ where $\bar{X}_{t}:=\sup _{s \leq t} X_{s}$. In that case the controlled process $U_{t}^{a}=X_{t}-L_{t}^{a}$ is a spectrally negative Lévy process reflected in the barrier $a$.

This result has been re-considered very recently in [3] for Cramér-Lundberg processes with a general jump distribution. In the latter paper it was shown that for an appropriate choice of jump distribution, the above described barrier strategy is not optimal. In much greater generality, the paper [2] focuses on the spectrally negative case and finds sufficient conditions for the optimal strategy to consist of a simple barrier strategy. It is in the latter paper that we first begin to see the connection with scale functions as the sufficient conditions given in [2] are phrased in terms of a variational inequality involving the value of a barrier strategy which itself can be expressed in terms of the associated scale function $W^{(q)}$. In a remarkable development shortly thereafter, Loeffen [26] made a decisive statement connecting the shape of the scale function $W^{(q)}$ to the existence of an optimal barrier strategy. Loeffen's result begins by requiring that the scale function $W^{(q)}$ is sufficiently smooth meaning that it belongs to $C^{1}(0, \infty)$ if $X$ is of bounded variation and otherwise belongs to $C^{2}(0, \infty)$. Loeffen's theorem reads as follows. 
Theorem 1.1. Suppose that $X$ is such that its scale functions are sufficiently smooth. Let

$$
a^{*}=\sup \left\{a \geq 0: W^{(q) \prime}(a) \leq W^{(q) \prime}(x) \text { for all } x \geq 0\right\},
$$

(which is necessarily finite) where we understand $W^{(q)^{\prime}}(0)=W^{(q)^{\prime}}(0+)$. Then the barrier strategy at $a^{*}$ is an optimal strategy if

$$
W^{(q) \prime}(a) \leq W^{(q) \prime}(b) \text { for all } a^{*} \leq a \leq b<\infty .
$$

The condition (1.4) is tantamount to saying that the scale function $W^{(q)}$ is convex beyond the global minimum of its first derivative. An intriguing result in itself, it is however arguably not a particularly practical condition to verify. None-the-less [26] makes one further striking step by providing a very natural class of Lévy risk processes for which (1.4) holds. More

precisely, it is shown that (1.4) holds when the Lévy measure $\Pi$ is absolutely continuous with a completely monotone density.

Thanks then to Theorem 1.1 a clear mandate is set with regard to finding as broad a class of Lévy processes as possible for which the barrier strategy is optimal through smoothness and convexity properties of the scale functions $W^{(q)}$. Motivated by this problem this paper serves a twofold purpose. Firstly to establish results which discuss the issue of smoothness and convexity of scale functions and secondly, using some of the latter results, to return to de Finetti's control problem and establish a larger class of Lévy processes for which the barrier strategy is optimal.

The remainder of the paper is structured as follows. In Section 2 we present an ensemble of results which provide sufficient conditions for smoothness, concavity and (ultimate) convexity of scale functions. Key to some of the results in this section are recent potential analytic developments in the theory of subordinators. In Section 3 we give our main result on de Finetti's control problem: when the Lévy measure of the underlying process has a log convex density, the solution to de Finetti's control problem is a barrier strategy. We then make a few remarks about this result and the main issues involved in the proof of this result. Also in this section we explain why this is a broader class of Lévy processes by giving some explicit examples. In Section 4 we use the results of Section 2 to prove our main result on de Finetti's control problem. We are not able to apply Theorem 1.1 verbatim for the present case however. Instead we must revisit its proof in order to weaken the meaning of 'sufficiently smooth' in its statement. Ultimately this requires the involvement of stochastic calculus which appeals to both semi-martingale local time and Markov local time. Some of the proofs of Section 2 are left to an Appendix.

\section{Convexity and Smoothness of Scale Functions}

We will first deal with 0-scale functions for spectrally negative Lévy processes that do not drift to $-\infty$, that is, processes for which $\Phi(0)=0$. Unless otherwise stated throughout this 
section we will assume that the measure $\Pi$ has a strictly positive density $\pi(x), x>0$, with respect to the Lebesgue measure. In this case,

$$
\Upsilon(x)=\Pi(x, \infty):=\bar{\Pi}(x)=\int_{x}^{\infty} \pi(y) d y, \quad x>0 .
$$

Before stating our first result we recall that a subordinator $H$ is said special if there exists another subordinator $H^{*}$, the so-called conjugate, such that if $h$ and $h^{*}$ are their respective Laplace exponents then

$$
\theta=h(\theta) h^{*}(\theta), \quad \theta \geq 0
$$

We refer to [32] for a recent account of properties of this subclass of subordinators. We also mention here in particular that the identification of this class of subordinators has permitted quite significant developments in their potential analysis. Indeed it is the latter developments which play a significant role in the forthcoming analysis of scale functions.

Our first result is on the concavity of the 0-scale function which, for convenience, we henceforth denote by $W$ instead of $W^{(0)}$.

Theorem 2.1. Assume that $\Phi(0)=0$. If the function $x \mapsto \bar{\Upsilon}(x):=\int_{x}^{\infty} \Upsilon(z) d z$ is log convex on $(0, \infty)$, then the scale function $W$ is concave on $(0, \infty)$.

Proof. It follows from the log convexity of $\bar{\Upsilon}$ and Theorem 2.4 of 32 that $\widehat{H}$ is a special subordinator and the renewal function of $\widehat{H}$ has a decreasing derivative $u$ which is also called the potential density of $\widehat{H}$. Since $W^{\prime}(x)=u(x)$, we know that $W$ is concave.

The next theorem is one of our main results of this section.

Theorem 2.2. Assume that $\Phi(0)=0$. If the function $x \mapsto \bar{\Pi}(x)=\int_{x}^{\infty} \pi(s) d s$ is log convex on $(0, \infty)$, then the function $W^{\prime}$ is convex on $(0, \infty)$. Furthermore, if $X$ has a Gaussian term or equivalently the drift of the descending ladder height process is strictly positive then $W \in C^{2}(0, \infty)$.

Proof. By our assumption, we know that the function

$$
\bar{\Upsilon}(x)=\int_{x}^{\infty} \bar{\Pi}(s) d s, \quad x>0
$$

is in $C^{1}(0, \infty)$. It follows from the first paragraph in the proof of Theorem 2 in [14] that this function is also log convex. Therefore it follows from Theorem 2.4 of [32] that $\widehat{H}$ is a special subordinator and the renewal function of $\widehat{H}$ has a decreasing derivative $u$ which is also called the potential density of $\widehat{H}$. It follows from [30] that the function $u$ satisfies the following equation

$$
\mathrm{d} u(t)+\int_{0}^{t} \bar{\Upsilon}(t-s) u(s) d s=1, \quad t>0
$$


where $\mathrm{d} \geq 0$ is the drift of $\widehat{H}$. Now when $\mathrm{d}=0$ we can apply Theorem 3 of [15] to conclude that the function $u$ is convex. When $\mathrm{d}>0$ we can apply Theorem 2 of [14] combined with the first two sentences of Section 4 in [14], to conclude that $u$ is convex and in $C^{1}(0, \infty)$. Now the conclusion follows since $W^{\prime}(x)=u(x)$.

The two theorems above and the arguments used in their proofs have several consequences, the first of which can be summarized as follows. If $\bar{\Pi}(s), s>0$, is a log convex function, then $\bar{\Upsilon}(s), s>0$, is also log convex and $W^{\prime}$ is a decreasing and convex function which implies that the subordinator $\widehat{H}$ with the tail of its Lévy measure given by $\bar{\Upsilon}$ is special, and thus there exists a subordinator $\widehat{H}^{*}$, with the tail of its Lévy measure denoted by $\bar{\Upsilon}^{*}(x)$, such that

$$
W^{\prime}(x)=W^{\prime}(\infty)+\bar{\Upsilon}^{*}(x), \quad x>0,
$$

and as a consequence $\Upsilon^{*}$ has a decreasing density in $(0, \infty)$. Then Theorem 2 and Corollary 1 in [25] imply in turn that there exists a spectrally negative Lévy process that does not drift to $-\infty$ such that its scale function $W^{*}$ satisfies

$$
W^{* \prime}(x)=\kappa+\bar{\Upsilon}(x), \quad x>0
$$

and therefore $W^{* \prime}$ is $\log$ convex.

Another interesting consequence provides a sufficient condition in terms of the potential density to guarantee that a subordinator has a Lévy measure with a decreasing density. This may be useful in the cases where a subordinator is characterized by its potential measure, as in the case of subordinators arising in the random covering of the positive reals, see e.g. [11].

Corollary 2.3. Let $H$ be a subordinator such that its potential measure has a density, say $W^{\prime}$, in $(0, \infty)$ such that $W^{\prime}$ is non-increasing and $-W^{\prime \prime}$ is non-increasing and log convex. Then the Lévy measure of $H$ has non-increasing density.

Proof. Since the potential measure of $H$ has a non-increasing density, we know that $H$ is a special subordinator whose conjugate we will denote by $H^{*}$. Furthermore, the tail of the Lévy measure of $H^{*}$ equals $W^{\prime}(x)-W^{\prime}(\infty), x>0$, and then its density is given by $-W^{\prime \prime}$. We now argue as in Theorem 2.2 to ensure that the potential measure of $H^{*}$ admits a decreasing and convex density in $(0, \infty)$. This finishes the proof since the tail of the Lévy measure of $H$ equals the density in $(0, \infty)$ of the potential measure of $H^{*}$.

An interesting question is whether a given function is the scale function of a spectrally negative Lévy process. It has been proved in Corollary 2 in 25] that a sufficient condition is that such a function is a Bernstein function. In the next result we provide a weaker sufficient condition. 
Corollary 2.4. Suppose that $W$ is a function on $\mathbb{R}$ such that $W(x)=0$ for all $x<0$ and that $W$ is positive and continuous on $[0, \infty)$. If $W$ is a concave non-decreasing function on $[0, \infty)$ such that $W^{\prime}$ is non-increasing on $(0, \infty)$ with $a:=\lim _{x \downarrow 0} x W^{\prime}(x)<\infty$ and $-W^{\prime \prime}$ is non-increasing and log convex on $(0, \infty)$, then there exists a spectrally negative Lévy process such that $W$ is its 0 -scale function.

Proof. We claim that $-W^{\prime \prime}$ is the Lévy density of some subordinator, that is,

$$
-\int_{0}^{\infty}(1 \wedge x) W^{\prime \prime}(x) d x<\infty
$$

In fact, since $W^{\prime}$ is non-increasing, we have

$$
-\int_{1}^{\infty} W^{\prime \prime}(x) d x=W^{\prime}(1)-W^{\prime}(\infty)<\infty
$$

On the other hand, since $W^{\prime \prime}$ is non-decreasing, we have for any $x \in(0,1)$,

$$
\begin{aligned}
-\int_{x}^{1} y W^{\prime \prime}(y) d y & =-\int_{x}^{1} y d W^{\prime}(y)=x W^{\prime}(x)-W^{\prime}(1)+\int_{x}^{1} W^{\prime}(y) d y \\
& =x W^{\prime}(x)-W^{\prime}(1)+W(1)-W(x) \\
& \leq W(1)-W(0)-W^{\prime}(1)+\lim _{x \downarrow 0} x W^{\prime}(x) \\
& \leq W(1)-W(0)-W^{\prime}(1)+a .
\end{aligned}
$$

Thus the claim is valid.

We may now deduce that $-W^{\prime \prime}$ is the Lévy density of some special subordinator $H^{*}$, whose conjugate, $H$, admits $W$ as its potential measure. By Corollary 2.3 the Lévy measure of $H$ has a non-increasing density. Thus we can construct a spectrally negative Lévy process $X$ whose descending ladder height process is $H$, see for example [17], which also satisfies the assertions of Corollary 2.4.

As we mentioned before the conditions in Corollary 2.4 are weaker than those in Corollary 2 in [25], because every Bernstein function $f$ is a non-decreasing concave function, and $f^{\prime}$ and $-f^{\prime \prime}$ are completely monotone functions with $\lim _{x \downarrow 0} x f^{\prime}(x)=0$.

Corollary 2.5. Let $H$ be a subordinator whose Lévy density, say $\Upsilon(x), x>0$, is log convex (and hence non-increasing) then the restriction of the potential measure to $(0, \infty)$ has a nonincreasing and convex density. If furthermore, the drift of $H$ is strictly positive then the density is in $C^{1}(0, \infty)$. 
Proof. As above, using the arguments in the first paragraph in the proof of Theorem 2 in [14] we get that $\bar{\Upsilon}(x)=\int_{x}^{\infty} \Upsilon(y) d y, x>0$, is log convex. Then by Theorem 2.4 in [32] we know that $H$ is a special subordinator and therefore the restriction of its potential measure to $(0, \infty)$ has a non-increasing density. Now, we can simply repeat the arguments in the proof of Theorem 2.2 to obtain the convexity and $C^{1}(0, \infty)$ result.

The following result is the analogue of Theorems 2.1 and 2.2 for $q$-scale functions for $q>0$ if $\Phi(0)=0$ and $q \geq 0$ if $\Phi(0)>0$.

Theorem 2.6. If the function

$$
\bar{\Pi}(x):=\int_{x}^{\infty} \pi(s) d s, \quad x>0
$$

is $\log$ convex, then for any $q>0$ if $\Phi(0)=0$, and $q \geq 0$ if $\Phi(0)>0$, the function $g_{q}(x):=$ $e^{-\Phi(q) x} W^{(q)}(x), x>0$, is concave. If furthermore, the function $\pi$ is log convex (and hence non-increasing) then $\bar{\Pi}(x)$ is log convex, the first derivative of $g_{q}$ is non-increasing and convex and the functions $W^{(q)}$ and $W^{(q) \prime}$ are strictly convex in the interval $\left(a^{*}, \infty\right)$, where

$$
a^{*}=\sup \left\{a \geq 0: W^{(q)^{\prime}}(a) \leq W^{(q) \prime}(y) \text { for all } y \geq 0\right\}<\infty \text {. }
$$

Finally, if the latter assumption is satisfied and the Gaussian coefficient is strictly positive then $W^{(q)} \in C^{2}(0, \infty)$.

The proof of this theorem relies on the following technical lemmas. Their proofs will be postponed to the Appendix. Note that in the first lemma below, the term $q / \Phi(q)$ is to be understood in the limiting sense, namely $\psi^{\prime}(0+)$, when $q=0$ and $\Phi(0)=0$.

Lemma 2.7. For each $q \geq 0$, the function $\widehat{\kappa}(q, \cdot)$ is a Bernstein function and its killing term is given by

$$
\widehat{\kappa}(q, 0)=\frac{q}{\Phi(q)}
$$

its drift term is given by

$$
\lim _{\theta \rightarrow \infty} \frac{\widehat{\kappa}(q, \theta)}{\theta}=\mathrm{d}
$$

and the tail of its Lévy measure is given by

$$
\bar{\Upsilon}_{q}(x):=e^{\Phi(q) x} \int_{x}^{\infty} e^{-\Phi(q) y} \bar{\Pi}(y) d y, \quad x>0
$$

Furthermore, if $\pi$ is non-increasing then for $q \geq 0$, the Lévy density associated to $\widehat{\kappa}(q, \cdot)$ is non-increasing. 
Lemma 2.8. If $\pi$ is log convex (and hence non-increasing), then for every $q \geq 0$ the function

$$
\bar{\Pi}(x)-\Phi(q) e^{\Phi(q) x} \int_{x}^{\infty} e^{-\Phi(q) y} \bar{\Pi}(y) d y, \quad x>0,
$$

is log convex.

Proof of Theorem 2.6. We have by assumption that the function $\bar{\Pi}$ is $\log$ convex, which implies that $e^{-\Phi(q) x} \bar{\Pi}(x) x>0$ is also log convex. Hence it follows from the first paragraph in the proof of Theorem 2 in [14] that the latter implies that the functions

$$
\int_{x}^{\infty} e^{-\Phi(q) s} \bar{\Pi}(s) d s, \quad e^{\Phi(q) x} \int_{x}^{\infty} e^{-\Phi(q) s} \bar{\Pi}(s) d s \quad x>0,
$$

are log convex. It follows that the function $\bar{\Upsilon}_{q}$ as defined in Lemma 2.7 is log convex and thus by Theorem 2.4 in [32] we have that the potential density associated to the Bernstein function $\widehat{\kappa}(q, \cdot)$ has a non-increasing density in $(0, \infty)$ that we will denote by $u_{q}$. It follows from Lemmas 1 and 2 in [25] that the function $\widehat{\kappa}(q, \Phi(q)+\cdot)$ still is a Bernstein function such that its potential measure admits the function $e^{-\Phi(q) x} u_{q}(x)$ as its density in $(0, \infty)$. It now follows that the later function is non-increasing and $\lim _{x \rightarrow \infty} e^{-\Phi(q) x} u_{q}(x)=0$.

It is well known that the $q$-scale function $W^{(q)}$ satisfies $W^{(q)}(x)=e^{\Phi(q) x} W_{\Phi(q)}(x), x>0$, where $W_{\Phi(q)}$ is the 0-scale function of the spectrally negative Lévy process with Laplace exponent given by $\psi_{q}(\theta)=\psi(\theta+\Phi(q))-q, \theta \geq 0$, see e.g. Lemma 8.4 in [20] for a proof of this fact. By the Wiener-Hopf factorization we have that $\psi_{q}$ is given by

$$
\psi_{q}(\theta)=\theta \widehat{\kappa}(q, \Phi(q)+\theta), \quad \theta \geq 0 .
$$

This implies in turn that

$$
\frac{\theta}{\psi_{q}(\theta)}=\frac{1}{\widehat{\kappa}(q, \Phi(q)+\theta)}=\mathrm{d}_{q}^{*}+\int_{0}^{\infty} e^{-\theta x} e^{-\Phi(q) x} u_{q}(x) d x, \quad \theta \geq 0,
$$

where $\mathrm{d}_{q}^{*}=\lim _{\theta \rightarrow \infty} 1 / \widehat{\kappa}(q, \Phi(q)+\theta) \geq 0$. By the definition of 0-scale functions and integration by parts in the latter equation it follows that

$$
\frac{1}{\psi_{q}(\theta)}=\int_{0}^{\infty} e^{-\theta x} W_{\Phi(q)}(x) d x=\int_{0}^{\infty} e^{-\theta x}\left(\mathrm{~d}_{q}^{*}+\int_{0}^{x} e^{-\Phi(q) z} u_{q}(z) d z\right) d x, \quad \theta \geq 0 .
$$

Thus the uniqueness of the Laplace transform implies that

$$
W_{\Phi(q)}(x)=\mathrm{d}_{q}^{*}+\int_{0}^{x} e^{-\Phi(q) z} u_{q}(z) d z, \quad x \geq 0 .
$$

Now the first claim immediately follows. The claim about $\bar{\Pi}$ is proved in the proof of Lemma 2.8. To prove the third claim we recall that under the assumption that $\pi$ is $\log$ 
convex (and hence non-increasing) Lemmas 2.7 and 2.8 imply that the Lévy density of the Bernstein function $\widehat{\kappa}(q, \cdot)$ is log convex (and hence non-increasing). Hence the hypotheses of Corollary 2.5 are satisfied and therefore $u_{q}$ is a non-increasing convex function and, whenever the Gaussian coefficient, equivalently the linear term in $\widehat{\kappa}$, is strictly positive we have $u_{q} \in$ $C^{1}(0, \infty)$. By elementary arguments it follows that $e^{-\Phi(q) x} u_{q}(x), x>0$, satisfies the same properties. Thus $g_{q}$ is a concave function whose first derivative is convex and continuous in $(0, \infty)$.

To prove the claim about the convexity of $W^{(q)}$ and $W^{(q) \prime}$, we observe that as $W^{(q) \prime}$ is given by

$$
W^{(q) \prime}(x)=\Phi(q) W^{(q)}(x)+u_{q}(x), \quad x>0 ;
$$

and since $u_{q}$ is convex, we will automatically get that $W^{(q) \prime}$ is ultimately convex once we have proved that $W^{(q)}$ is ultimately convex. Indeed, we have that

$$
W^{(q) \prime \prime}(x)=(\Phi(q))^{2} W^{(q)}(x)+\Phi(q) u_{q}(x)+u_{q}^{\prime}(x), \quad \text { a.e. } x>0 .
$$

Then as $u_{q}^{\prime}$ increases and $W^{(q)}$ grows exponentially fast it follows that ultimately $W^{(q) \prime \prime}>0$. Hence $W^{(q)}$ and $W^{(q) \prime}$ are ultimately strictly convex. Furthermore, because $W^{(q) \prime}$ tends to infinity as $x$ tends to $\infty$, it follows that $a^{*}<\infty$. Now, let $\alpha_{1}<\alpha_{2}$ be points at which $W^{(q) \prime}$ reaches a local minimum. Because of the convexity of $u_{q}$ we know that the right and left derivatives of $u_{q}$ exist everywhere and they satisfy that $u_{q}^{\prime-}\left(\alpha_{1}\right) \leq u_{q}^{\prime+}\left(\alpha_{1}\right) \leq u_{q}^{\prime-}\left(\alpha_{2}\right) \leq$ $u_{q}^{\prime+}\left(\alpha_{2}\right)$. As a consequence the right and left derivatives of $W^{(q) \prime}$ exist everywhere and satisfy

$$
W^{(q) \prime \prime-}\left(\alpha_{i}\right)=\Phi(q) W^{(q) \prime}\left(\alpha_{i}\right)+u_{q}^{\prime-}\left(\alpha_{i}\right) \leq 0, \quad W^{(q) \prime \prime+}\left(\alpha_{i}\right)=\Phi(q) W^{(q) \prime}\left(\alpha_{i}\right)+u_{q}^{\prime+}\left(\alpha_{i}\right) \geq 0,
$$

for $i=1,2$. These facts together imply that

$$
0 \leq \Phi(q)\left(W^{(q) \prime}\left(\alpha_{1}\right)-W^{(q) \prime}\left(\alpha_{2}\right)\right)+u_{q}^{\prime+}\left(\alpha_{1}\right)-u_{q}^{\prime-}\left(\alpha_{2}\right),
$$

and hence $W^{(q) \prime}\left(\alpha_{1}\right)-W^{(q) \prime}\left(\alpha_{2}\right) \geq 0$. This implies that the last place where $W^{(q) \prime}$ reaches a local minimum is also the last place where it hits its global minimum. Moreover, for $x>0$ we have that $W^{(q) \prime}\left(a^{*}\right) \leq W^{(q) \prime}(x)$. It thus follows that the following inequalities

$$
0 \leq \Phi(q) W^{(q) \prime}\left(a^{*}\right)+u_{q}^{\prime+}\left(a^{*}\right) \leq \Phi(q) W^{(q) \prime}(x)+u_{q}^{\prime-}(x) \leq \Phi(q) W^{(q) \prime}(x)+u_{q}^{\prime+}(x),
$$

hold for $x>a^{*}$. Actually, the second inequality is a strict one. Indeed, if there would exist $x^{*}>a^{*}$ such that $\Phi(q) W^{(q) \prime}\left(a^{*}\right)+u_{q}^{\prime+}\left(a^{*}\right)=\Phi(q) W^{(q) \prime}\left(x^{*}\right)+u_{q}^{\prime-}\left(x^{*}\right)$, then since $u_{q}^{\prime-}\left(x^{*}\right)-$ $u_{q}^{\prime+}\left(a^{*}\right) \geq 0$, we would have that $W^{(q) \prime}\left(a^{*}\right) \geq W^{(q) \prime}\left(x^{*}\right)$, which would be a contradiction to the fact that $a^{*}$ is the largest value where $W^{(q) \prime}$ attains its global minimum. It follows that $W^{(q) \prime}$ is strictly increasing for $x>a^{*}$. That is $W^{(q)}$ is strictly convex in $\left(a^{*}, \infty\right)$ and, from equation (2.2), we deduce that $W^{(q) \prime}$ is also strictly convex for $x>a^{*}$. Finally, the equation (2.3) proves also that when furthermore the Gaussian coefficient is strictly positive then $W^{(q)} \in C^{2}(0, \infty)$ as in this case we already proved that $u_{q} \in C^{1}(0, \infty)$. 
We now leave behind the assumption that $\Pi$ has a strictly positive density and allow $\Pi$ to be any Lévy measure. The following result provides necessary and sufficient conditions for a scale function associated to a spectrally negative Lévy process of bounded variation to be in the class $C^{1}(0, \infty)$.

Theorem 2.9. Assume that $X$ is a spectrally negative Lévy process of bounded variation. The following conditions are equivalent

(i) $W \in C^{1}(0, \infty)$;

(ii) $W^{(q)} \in C^{1}(0, \infty)$ for all $q \geq 0$;

(iii) $\bar{\Pi} \in C^{0}(0, \infty)$.

Proof. First we prove that (i) and (iii) are equivalent. As $X$ is assumed to be of bounded variation it follows that $X_{t}=\delta t-S_{t}$, where $\delta>0$ and $S$ is a subordinator. Let $\bar{n}$ be the excursion measure of $X$ reflected at its supremum. For background on the excursion theory of Lévy processes reflected at their supremum, see e.g. [4], 9], [20]. A standard result, see e.g. Lemma 8.2 in [20], says that $W \in C^{1}(0, \infty)$ if and only if the law of the height of the excursion process has no atoms, that is, the measure

$$
\bar{n}\left(\sup _{0 \leq s \leq \zeta} \epsilon(s) \in d x\right), \quad x>0
$$

has no atoms; where $\epsilon$ and $\zeta$ denotes the generic excursion process and its lifetime respectively. The proof of the equivalence of (i) and (iii) will be obtained as a consequence of the fact above and the identity

$$
\bar{n}\left(\sup _{0 \leq s \leq \zeta} \epsilon(s)>z\right)=\frac{1}{\delta} \Pi(z, \infty)+\frac{1}{\delta} \int_{0}^{z} \Pi(d x)\left(1-\frac{W(z-x)}{W(z)}\right), \quad z>0 .
$$

If we take this identity for granted, then we have that

$$
\bar{n}\left(\sup _{0 \leq s \leq z} \epsilon(s)=z\right)=\frac{1}{\delta} \Pi\{z\}+\frac{1}{\delta} \Pi\{z\}\left(1-\frac{W(0)}{W(z)}\right), \quad z>0 .
$$

It follows then that $\bar{n}\left(\sup _{0 \leq s \leq \zeta} \epsilon(s) \in d x\right)$ has atoms if and only if $\Pi$ does. This proves the equivalence of (i) and (iii).

We will now prove the identity (2.4). It is known from [29] and Proposition 5 in [33] that when $X$ is of bounded variation the excursion measure of $X$ reflected at its supremum can be described by the formula

$$
\bar{n}(F(\epsilon(s), 0 \leq s \leq \zeta))=\frac{1}{\delta} \int_{0}^{\infty} \Pi(d x) \widehat{\mathbb{E}}_{x}\left(F\left(X_{s}, 0 \leq s \leq \tau_{0}^{-}\right)\right),
$$


where $F$ is any nonnegative measurable functional on the space of cadlag paths, $\widehat{\mathbb{E}}_{x}$ denotes the law of the dual Lévy process $\widehat{X}=-X$ and $\tau_{x}^{-}=\inf \left\{s>0: X_{s}<x\right\}, x \in \mathbb{R}$. We will also denote by $\tau_{z}^{+}=\inf \left\{s>0: X_{s}>z\right\}, z \in \mathbb{R}$. Hence, it follows that

$$
\begin{aligned}
\bar{n}\left(\sup _{0 \leq s \leq \zeta} \epsilon(s)>z\right) & =\frac{1}{\delta} \int_{0}^{\infty} \Pi(d x) \widehat{\mathbb{P}}_{x}\left(\sup _{0 \leq s \leq \tau_{0}^{-}} X_{s}>z\right) \\
& =\frac{1}{\delta} \Pi(z, \infty)+\frac{1}{\delta} \int_{0}^{z} \Pi(d x) \widehat{\mathbb{P}}_{x}\left(\tau_{z}^{+}<\tau_{0}^{-}\right) \\
& =\frac{1}{\delta} \Pi(z, \infty)+\frac{1}{\delta} \int_{0}^{z} \Pi(d x) \widehat{\mathbb{P}}\left(\tau_{z-x}^{+}<\tau_{-x}^{-}\right) \\
& =\frac{1}{\delta} \Pi(z, \infty)+\frac{1}{\delta} \int_{0}^{z} \Pi(d x) \mathbb{P}\left(\tau_{x-z}^{-}<\tau_{x}^{+}\right) \\
& =\frac{1}{\delta} \Pi(z, \infty)+\frac{1}{\delta} \int_{0}^{z} \Pi(d x)\left(1-\frac{W(z-x)}{W(z)}\right)
\end{aligned}
$$

where in the last equality we have used Takac's solution to the two sided exit problem for spectrally negative Lévy processes, see e.g. [4] Theorem VII.8.

To complete the proof we show that (ii) and (iii) are equivalent. There is very little work to do as soon as one recalls the fact stated earlier that for $q>0$

$$
W^{(q)}(x)=e^{\Phi(q) x} W_{\Phi(q)}(x)
$$

where $W_{\Phi(q)}$ is the 0 -scale function of the spectrally negative Lévy process whose Laplace exponent is given by $\psi(\theta+\Phi(q))-q$. The latter process has a Lévy measure $\Pi_{\Phi(q)}$ given by $\Pi_{\Phi(q)}(d x)=e^{-\Phi(q) x} \Pi(d x)$ on $(0, \infty)$. It follows that $\Pi$ has no atoms if and only if $\Pi_{\Phi(q)}$ has no atoms. Consequently the arguments above leading to the equivalence of (i) and (iii) show that $W_{\Phi(q)}$ belongs to $C^{1}(0, \infty)$ or equivalently (ii) holds, if and only if (iii) holds.

Note that another proof of the last result may be implicitly extracted from Lemma 1 (iii) in [8]. In essence that would again necessitate the observation that the entrance law of excursions begin with a jump whose intensity is given by $\delta^{-1} \Pi$.

\section{De Finetti's control problem}

In this section we shall discuss an important consequence of Theorem 2.6 pertaining to de Finetti's control problem. In particular we shall prove the following result.

Theorem 3.1. Suppose that $X$ has a Lévy density $\pi$ that is log convex then the barrier strategy at $a^{*}$ is optimal for (1.3). 
Before proceeding to the proof of Theorem 3.1, let us first make some remarks.

1. In principle the proof of Theorem 3.1 follows directly from Theorem 2.6 and Theorem 1.1] if one can verify that $W^{(q)}$ is sufficiently smooth. This is possible in most cases, but not all. The outstanding case is the focus of the proof of Theorem 3.1 and we identify it below by excluding the cases for which sufficient smoothness can be established.

If $a^{*}=0$ then necessarily, either $\sigma>0$, or $\sigma=0$ and $\Pi(0, \infty)<\infty$ simultaneously. Other types of spectrally negative Lévy processes are not possible when $a^{*}=0$ since then necessarily $W^{(q)}(0+)=\infty$. In the case $\sigma>0$ we see that $W^{(q)} \in C^{2}(0, \infty)$ by Theorem 2.6 and when $\sigma=0$ and $\Pi(0, \infty)<\infty$ simultaneously we see from (2.2) $W^{(q)} \in C^{1}(0, \infty)$. Thus when $a^{*}=0$ we have that $W^{(q)}$ is sufficiently smooth.

Suppose now that $a^{*}>0$. If $X$ is of bounded variation or $\sigma>0$ then, similar to the previous paragraph we may again deduce from Theorem 2.6 and (2.2) that $W^{(q)}$ is sufficiently smooth.

The outstanding case is thus given by $a^{*}>0, X$ is of unbounded variation and $\sigma=0$.

2. Recall that Theorem 3 of [26] states that if $X$ has Lévy density $\pi$ which is completely monotone then $W^{(q) \prime}$ is convex on $(0, \infty)$ and hence the barrier strategy at $a^{*}$ is an optimal strategy. Theorem 3.1 is an improvement on this result on account of the fact that any completely monotone function density is log convex. Below are two some examples of Lévy densities which meet the criteria of Theorem 3.1 but not Theorem 3 of [26].

Suppose that $f$ and $g$ both map $(0, \infty)$ to $[0, \infty)$ and that they are both non-increasing and $\log$ convex. Suppose moreover that for some (and hence every) $\varepsilon>0, \int_{0}^{\varepsilon} x^{2} f(x) d x<$ $\infty$ and $\int_{\varepsilon}^{\infty} g(x) d x<\infty$. Further, for some fixed $\alpha>0$ we have $f(\alpha)=g(\alpha)$ and

$$
\frac{f^{\prime-}(\alpha)}{f(\alpha)} \leq \frac{g^{\prime+}(\alpha)}{g(\alpha)}
$$

Then

$$
\pi(x):= \begin{cases}f(x) & x \in(0, \alpha) \\ g(x) & x \in[\alpha, \infty)\end{cases}
$$

is an example of a decreasing, log convex function which is not completely monotone in general. Specific cases in which $\pi$ is not completely monotone may be taken to be

(i) $f(x)=x^{-\left(1+\lambda_{1}\right)}, g(x)=x^{-\left(1+\lambda_{2}\right)}, \alpha=1$ where $0<\lambda_{2}<\lambda_{1}<2$,

(ii) $f(x)=e^{2-x}, g(x)=e^{1-\lambda x}, \alpha=1 /(1-\lambda)$ where $0<\lambda<1$. 
3. It is also worth noting that if the Lévy density $\pi$ meets the conditions of Theorem 3.1 but is not completely monotone as in Theorem 3 of [26], then the behaviour of the scale function $W^{(q)}$ on $\left(0, a^{*}\right)$ is not necessarily concave as is the case in the aforementioned theorem.

4. The proof of Theorem 3.1, given in the next section, is lengthy requiring some auxiliary results first. Scanning the proof it is not immediately clear where the need for convexity on $\left(a^{*}, \infty\right)$ is needed. The precise point at which this property is required is embedded in the proof of Lemma 4.3 below and we have indicated as such in the proof.

\section{Proof of Theorem 3.1}

Following the first remark in the previous section, we shall assume throughout this section that $a^{*}>0, X$ is of unbounded variation and $\sigma=0$. Moreover we shall assume that the conditions of Theorem 3.1 are in force.

We define an operator $(\Gamma, \mathcal{D}(\Gamma))$ as follows. $\mathcal{D}(\Gamma)$ stands for the family of functions $f \in C^{1}(0, \infty)$ such that the integral

$$
\int_{(0, \infty)}\left[f(x-y)-f(x)+y f^{\prime}(x) \mathbf{1}_{\{y \leq 1\}}\right] \Pi(d y)
$$

is absolutely convergent for all $x>0$. For any $f \in \mathcal{D}(\Gamma)$, we define

$$
\Gamma f(x)=\gamma f^{\prime}(x)+\int_{(0, \infty)}\left[f(x-y)-f(x)+y f^{\prime}(x) \mathbf{1}_{\{y \leq 1\}}\right] \Pi(d y) .
$$

Recall that for any $a>0$, the expected value discounted at rate $q>0$ of the barrier strategy at level $a$ is given by

$$
v_{a}(x):=\mathbb{E}_{x}\left(\int_{\left[0, \sigma^{a}\right]} e^{-q t} d L_{t}^{a}\right)= \begin{cases}W^{(q)}(x) / W^{(q) \prime}(a), & -\infty<x \leq a \\ x-a+W^{(q)}(a) / W^{(q) \prime}(a), & \infty>x>a .\end{cases}
$$

where $\sigma^{a}=\inf \left\{t>0: U_{t}^{a}<0\right\}$. The second equality is taken from [2].

Lemma 4.1. For any $a>0, v_{a} \in \mathcal{D}(\Gamma)$. Furthermore, the function $x \mapsto \Gamma v_{a}(x)$ is continuous in $(0, a)$.

Proof. We have proved in Section 2 that $W^{(q)}$ is in $C^{1}(0, \infty)$, hence we know that $v_{a}$ is in $C^{1}(0, \infty)$. To show that $v_{a} \in \mathcal{D}(\Gamma)$, we only need to show that the integral in the definition of $\Gamma v_{a}$ is absolutely convergent for all $x>0$. It is easy to check that this is true for $x>a$, 
so we are going to concentrate on $x \in(0, a)$. Note that it suffices to consider $W^{(q)}$ instead of $v_{a}$. For each $x \in(0, a)$ we may write the integral in the definition of $\Gamma W^{(q)}$ as

$$
\begin{aligned}
& \int_{(\varepsilon, \infty)}\left(W^{(q)}(x-y)-W^{(q)}(x)+y W^{(q) \prime}(x) \mathbf{1}_{\{y \leq 1\}}\right) \Pi(d y) \\
& +\int_{(0, \varepsilon)}\left(W^{(q)}(x-y)-W^{(q)}(x)+y W^{(q) \prime}(x)\right) \Pi(d y)
\end{aligned}
$$

where the value of $\varepsilon=\varepsilon(x) \in(0,1)$ is chosen for each $x$ such that $x-2 \varepsilon>0$. The absolute convergence of the first integral as well as its continuity in $x$ follows in a straightforward way as a consequence of the continuity and boundedness of $W^{(q) \prime}$ on bounded intervals and dominated convergence in the case of continuity. With regard to the second integral, recall that $W^{(q) \prime}(x)=\Phi(q) W^{(q)}(x)+u_{q}(x)$. Using the mean value theorem and the fact that $u_{q}$ is convex and decreasing, we get that for all $y \in(0, \varepsilon)$

$$
\begin{aligned}
& \left|W^{(q)}(x-y)-W^{(q)}(x)+y W^{(q) \prime}(x)\right| \\
& =y\left|W^{(q) \prime}(x)-W^{(q) \prime}(x-\xi(y))\right| \quad \text { where } \xi(y) \in(0, y) \\
& \leq \Phi(q) y\left|W^{(q)}(x)-W^{(q)}(x-\xi(y))\right|+y\left|u_{q}(x)-u_{q}(x-\xi(y))\right| \\
& \leq \Phi(q) y^{2} \sup _{z \in[-\varepsilon, \varepsilon]} W^{(q) \prime}(x+z)+y \int_{x-\xi(y)}^{x}\left|u_{q}^{\prime}(y)\right| d y \\
& \leq \Phi(q) y^{2} \sup _{z \in[-\varepsilon, \varepsilon]} W^{(q) \prime}(x+z)+y^{2}\left|u_{q}^{\prime}(x-\varepsilon)\right| \\
& \leq y^{2} \sup _{z \in[-\varepsilon, \varepsilon]}\left(\Phi(q) W^{(q) \prime}(x+z)+\left|u_{q}^{\prime}(x+z)\right|\right) .
\end{aligned}
$$

This estimate shows both that the second integral is uniformly integrable in (4.1) and continuous in $x$ by dominated convergence.

Lemma 4.2. For any $a>0$ we have

$$
(\Gamma-q) v_{a}(x)=0, \quad x \in(0, a) .
$$

Proof. It is well known that $e^{-q\left(t \wedge \tau_{a}^{+} \wedge \tau_{0}^{-}\right)} W^{(q)}\left(X_{t \wedge \tau_{a}^{+} \wedge \tau_{0}^{-}}\right)$is a $\mathbb{P}_{x^{-}}$martingale for each $x \in(0, a)$ (cf. [1]), thus $e^{-q\left(t \wedge \tau_{a}^{+} \wedge \tau_{0}^{-}\right)} v_{a}\left(X_{t \wedge \tau_{a}^{+} \wedge \tau_{0}^{-}}\right)$is a $\mathbb{P}_{x^{-}}$martingale for each $x \in(0, a)$. Appealing to the Meyer-Itô formula (cf. Theorem 70 of [27]) we have on $\left\{t<\tau_{a}^{+} \wedge \tau_{1 / n}^{-}\right\}$

$$
v_{a}\left(X_{t}\right)-v_{a}(x)=m_{t}+\int_{0}^{t} \Gamma v_{a}\left(X_{s}\right) d s+\int_{\mathbb{R}} v_{a}^{\prime \prime}(y) \ell(y, t) d y \quad \mathbb{P}_{x^{-}} \text {a.s. }
$$


where $\ell(y, \cdot)$ is the semi-martingale local time at $y$ of $X$ and, with $X^{(1)}$ as the martingale part of $X$ consisting of compensated jumps of size less than or equal to unity,

$$
\begin{aligned}
m_{t}= & \sum_{s \leq t}\left[\Delta v_{a}\left(X_{s}\right)-\Delta X_{s} v_{a}^{\prime}\left(X_{s-}\right) \mathbf{1}_{\left\{\left|\Delta X_{s}\right| \leq 1\right\}}\right] \\
& -\int_{0}^{t} \int_{(0, \infty)}\left[v_{a}\left(X_{s-}-y\right)-v_{a}\left(X_{s-}\right)+y v_{a}^{\prime}\left(X_{s-}\right) \mathbf{1}_{\{y \leq 1\}}\right] \Pi(d y) d s \\
& +\int_{0}^{t} v_{a}^{\prime}\left(X_{s-}\right) d X_{s}^{(1)}
\end{aligned}
$$

is a local martingale which is also a true martingale on account of the fact that $W^{(q) \prime}$ is bounded on $[1 / n, a]$. Note that we have used that the integral part of $\Gamma v_{a}(y)$ is absolutely convergent for each $y \in(0, a)$ in order to meaningfully write down the compensation in the expression for the martingale $m_{t}$. The occupation formula for the semimartingale local time of $X$ says that

$$
\int_{\mathbb{R}} \ell(y, t) g(y) d y=\sigma^{2} \int_{0}^{t} g\left(X_{s}\right) d s \quad \mathbb{P}_{x^{-}} \text {a.s. }
$$

where $g$ is a bounded Borel measurable function. This implies that for Lebesgue almost every $y, \ell(y, \cdot)$ is identically zero almost surely. Taking this into account the last integral in (4.2) is almost surely zero. Using the semi-martingale decomposition in (4.2), one may now use stochastic integration by parts for semi-martingales to deduce that on $\left\{t<\tau_{a}^{+} \wedge \tau_{1 / n}^{-}\right\}$

$$
e^{-q t} v_{a}\left(X_{t}\right)-v_{a}(x)=\lambda_{t}+\int_{0}^{t} e^{-q s}(\Gamma-q) v_{a}\left(X_{s}\right) d s \quad \mathbb{P}_{x^{-a . s .}}
$$

where $\lambda_{t}=\int_{0}^{t} e^{-q s} d m_{s}$ is a martingale.

Next, use uniform boundedness of $(\Gamma-q) v_{a}(x)$ on $(\alpha, \beta) \subset[0, a]$ and the martingale property of $e^{-q\left(t \wedge \tau_{\beta}^{+} \wedge \tau_{\alpha}^{-}\right)} v_{a}\left(X_{\left.t \wedge \tau_{\beta}^{+} \wedge \tau_{\alpha}^{-}\right)}\right.$to get

$$
0=\mathbb{E}_{x}\left[\int_{0}^{\tau_{\beta}^{+} \wedge \tau_{\alpha}^{-}} e^{-q s}(\Gamma-q) v_{a}\left(X_{s}\right) d s\right]=\int_{(\alpha, \beta)}(\Gamma-q) v_{a}(y) u^{(q)}(x, y) d y
$$

where $u^{(q)}(x, y) d y=\int_{0}^{\infty} e^{-q s} \mathbb{P}_{x}\left(X_{s} \in d y ; t<\tau_{\beta}^{+} \wedge \tau_{\alpha}^{-}\right)$is the strictly positive resolvent density of the process $X$ killed on exiting $(\alpha, \beta)$ (see [5] for more details). As $(\alpha, \beta)$ is arbitrary and $(\Gamma-q) v_{a}(x)$ is continuous, it follows that the latter is identically zero on $(0, a)$. This is due to a classical argument by contradiction. If the claim is false then there by continuity of $(\Gamma-q) v_{a}$ and strict positivity of $u^{(q)}$, there exists an interval $\left(\alpha^{\prime}, \beta^{\prime}\right) \subset[0, a]$ such that (without loss of generality) $(\Gamma-q) v_{a}(x)>0$ on $\left(\alpha^{\prime}, \beta^{\prime}\right)$. Then the equality (4.3) would be violated. 
For convenience, we use $v$ to denote the function $v_{a^{*}}, U$ to denote $U^{a^{*}}$ and $L$ to denote $L^{a^{*}}$. Then we have the following result.

Lemma 4.3. For any $x>0$ we have $(\Gamma-q) v(x) \leq 0$.

Proof. There is nothing to prove when $x \in\left(0, a^{*}\right)$ because of Lemma 4.2 applied to the case $a=a^{*}$. Thanks to the continuity given by Lemma 4.1, this maybe extended to $\left(0, a^{*}\right]$. Finally the inequality can be proved to hold on $\left(a^{*}, \infty\right)$ by following verbatim the arguments in the proof of Theorem 2 in [26], although it is not necessary to replicate the behaviour of second derivatives in that proof, since we have $\sigma=0$.

It is important to note that the use of the convexity of $W^{(q)}$ on $\left(a^{*}, \infty\right)$ appears in Theorem 2 of [26] and therefore in this paper the use of convexity is hidden in the latter part of the proof.

Now we are in a position to prove Theorem 3.1 .

Proof of Theorem 3.1. Recall that we are assuming that $a^{*}>0, X$ is of unbounded variation and $\sigma=0$. Also recall we use $v$ to denote the function $v_{a^{*}}, U$ to denote $U^{a^{*}}$ and $L$ to denote $L^{a^{*}}$. The idea of the proof are similar to that of [2] and [26], however it is necessary to revisit the main line of reasoning and provide more sensitive arguments that accommodate for the fact that in the present case $W^{(q)}$ is not sufficiently smooth, it is twice continuously differentiable almost everywhere but is not in $C^{2}(0, \infty)$.

Now let $\xi$ be an admissible strategy and let $\bar{L}^{\xi}$ be the cadlag modification of the process $L^{\xi}$. Note that it is still adapted as the usual conditions have been assumed on the natural filtration and hence $\bar{U}^{\xi}=X-\bar{L}^{\xi}$ is a semi-martingale. Since the integral in the definition of $\Gamma v$ is absolutely convergent for every $x>0$ and second derivative of $v$ is well defined Lebesgue almost everywhere, we can apply the Meyer-Itô formula (cf. Theorem 70 of [27]) to the process $v\left(\bar{U}^{\xi}\right)$ to get, after some straightforward manipulation, that on $\left\{t<\sigma^{\xi}\right\}$,

$$
\begin{aligned}
v\left(\bar{U}_{t}^{\xi}\right)-v\left(\bar{U}_{0}^{\xi}\right)= & M_{t}^{\xi}+\int_{0}^{t} \Gamma v\left(\bar{U}_{s-}^{\xi}\right) d s \\
& +\sum_{s \leq t} \mathbf{1}_{\left\{\Delta \bar{L}_{s}^{\xi}>0\right\}}\left\{v\left(\bar{U}_{s-}^{\xi}+\Delta X_{s}-\Delta \bar{L}_{s}^{\xi}\right)-v\left(\bar{U}_{s-}^{\xi}+\Delta X_{s}\right)\right\} \\
& -\int_{(0, t]} v^{\prime}\left(\bar{U}_{s-}^{\xi}\right) d \bar{L}_{s}^{\xi, c}+\frac{1}{2} \int_{\mathbb{R}} v^{\prime \prime}(x) \ell^{\xi}(x, t) d x
\end{aligned}
$$


such that

$$
\begin{aligned}
M_{t}^{\xi}= & \sum_{s \leq t} \mathbf{1}_{\left\{\left|\Delta X_{s}\right|>0\right\}}\left[v\left(\bar{U}_{s-}^{\xi}+\Delta X_{s}\right)-v\left(\bar{U}_{s-}^{\xi}\right)-\Delta X_{s} v^{\prime}\left(\bar{U}_{s-}^{\xi}\right) \mathbf{1}_{\left\{\left|\Delta X_{s}\right| \leq 1\right\}}\right] \\
& -\int_{0}^{t} \int_{(0, \infty)}\left[v\left(\bar{U}_{s-}^{\xi}-y\right)-v\left(\bar{U}_{s-}^{\xi}\right)+y v^{\prime}\left(\bar{U}_{s-}^{\xi}\right) \mathbf{1}_{\{y \leq 1\}}\right] \Pi(d y) d s \\
& +\int_{0}^{t} v^{\prime}\left(\bar{U}_{s-}^{\xi}\right) d X_{s}^{(1)}
\end{aligned}
$$

is a local martingale with $M_{0}^{\xi}=0$ where $X^{(1)}$ the martingale part of $X$ consisting of compensated jumps of size less than or equal to unity. Moreover $\bar{L}^{\xi, c}$ is the continuous part of $\bar{L}^{\xi}$ and $\ell^{\xi}(x, \cdot)$ is the semi-martingale local time at $x$ of $\bar{U}^{\xi}$. We have used in particular the absolute convergence of the integral part of $\Gamma v$ in order to make sense of $M_{t}^{\xi}$ as a compensated stochastic integral. Similarly to before, the occupation formula for the semimartingale local time of $\bar{U}^{\xi}$ reads

$$
\int_{\mathbb{R}} \ell^{\xi}(x, t) g(x) d x=\sigma^{2} \int_{0}^{t} g\left(\bar{U}_{s}^{\xi}\right) d s,
$$

where $g$ is a bounded Borel measurable function. Also similarly to before, since $\sigma=0$ this implies that for Lebesgue almost every $x, \ell^{\xi}(x, \cdot)$ is identically zero almost surely. Taking this into account the last integral in (4.4) is almost surely zero. Stochastic integration by parts now gives us on $\left\{t<\sigma^{\xi}\right\}$

$$
\begin{aligned}
e^{-q t} v\left(\bar{U}_{t}^{\xi}\right)-v\left(\bar{U}_{0}^{\xi}\right)= & \Lambda_{t}^{\xi}+\int_{0}^{t} e^{-q s}(\Gamma-q) v\left(\bar{U}_{s-}^{\xi}\right) d s \\
& +\sum_{s \leq t} \mathbf{1}_{\left\{\Delta \bar{L}_{s}^{\xi}>0\right\}} e^{-q s}\left\{v\left(\bar{U}_{s-}^{\xi}+\Delta X_{s}-\Delta \bar{L}_{s}^{\xi}\right)-v\left(\bar{U}_{s-}^{\xi}+\Delta X_{s}\right)\right\} \\
& -\int_{(0, t]} e^{-q s} v^{\prime}\left(\bar{U}_{s-}^{\xi}\right) d \bar{L}_{s}^{\xi, c},
\end{aligned}
$$

where $\Lambda_{t}^{\xi}=\int_{0}^{t} e^{-q s} d M_{s}^{\xi}$ is a local martingale.

Now note that by inspection, using the properties of $a^{*}$, we see $v^{\prime}(x) \geq 1$ and moreover, on $\left\{\Delta \bar{L}_{s}^{\xi}>0\right\}$,

$$
v\left(\bar{U}_{s-}^{\xi}+\Delta X_{s}-\Delta \bar{L}_{s}^{\xi}\right)-v\left(\bar{U}_{s-}^{\xi}+\Delta X_{s}\right)=-\int_{\bar{U}_{s-}^{\xi}+\Delta X_{s}-\Delta \bar{L}_{s}^{\xi}}^{\bar{U}_{s-}^{\xi}+\Delta X_{s}} v^{\prime}(x) d x \leq-\Delta \bar{L}_{s}^{\xi} .
$$

Note also that

$$
\int_{(0, t]} e^{-q s} d \bar{L}_{s}^{\xi}=\int_{[0, t]} e^{-q s} d L_{s}^{\xi}-L_{0+}^{\xi}
$$

and by the mean value theorem and the fact that $v^{\prime}(x) \geq 1$ we also have under $\mathbb{P}_{x}$ that $v\left(\bar{U}_{0}^{\xi}\right) \leq v(x)-L_{0+}^{\xi}$. Recalling the property that $(\Gamma-q) v \leq 0$ for all $x>0$ it follows that 
for any appropriate localization sequence of stopping times $\left\{T_{n}: n \geq 1\right\}$ we have under $\mathbb{P}_{x}$

$$
\begin{aligned}
v(x)-L_{0+}^{\xi} & \geq-\Lambda_{\sigma^{\xi} \wedge T_{n}}^{\xi}+\int_{\left(0, \sigma^{\xi} \wedge T_{n}\right]} e^{-q s} d \bar{L}_{s}^{\xi}+e^{-q\left(\sigma^{\xi} \wedge T_{n}\right)} v\left(\bar{U}_{\sigma^{\xi} \wedge T_{n}}^{\xi}\right) \\
& \geq-\Lambda_{\sigma^{\xi} \wedge T_{n}}^{\xi}+\int_{\left[0, \sigma \xi \wedge T_{n}\right]} e^{-q s} d L_{s}^{\xi}-L_{0+}^{\xi} .
\end{aligned}
$$

Taking expectation and then limits as $n \uparrow \infty$ and recalling that $\xi$ is an arbitrary strategy in $\Xi$, we thus deduce that

$$
v(x) \geq \sup _{\xi \in \Xi} \mathbb{E}_{x}\left(\int_{[0, \sigma \xi]} e^{-q t} d L_{t}^{\xi}\right)=v^{*}(x) .
$$

On the other hand, thanks to the expression

$$
v(x):=\mathbb{E}_{x}\left(\int_{\left[0, \sigma^{a^{*}}\right]} e^{-q t} d L_{t}^{a^{*}}\right),
$$

the upper bound is attained by the barrier strategy at $a^{*}$ and the proof is complete.

\section{Appendix: Proof of Lemmas 2.7 and 2.8}

Proof of Lemma 2.7. We recall that the right continuous inverse of the local time at 0 for $X$ reflected at its supremum, $L^{-1}$, and that of $X$ reflected at its infimum, say $\widehat{L}^{-1}$, are possibly killed subordinators whose Laplace exponents are given by $\Phi(\cdot)$, and $\widehat{\kappa}(\cdot, 0)$ respectively. It follows by the time-space Wiener-Hopf factorization that

$$
q=\Phi(q) \widehat{\kappa}(q, 0), \quad q \geq 0,
$$

see e.g. [4] Chapter VII. Thus $\widehat{\kappa}(q, 0)=q / \Phi(q), q \geq 0$. We recall that $\widehat{\kappa}(\cdot, \cdot)$ is the Laplace exponent of the bivariate descending ladder subordinator, and hence it can be represented as

$$
\widehat{\kappa}(\lambda, \beta)=\kappa(\lambda, 0)+\mathrm{d}_{1} \beta+\int_{(0, \infty)^{2}} \mu_{-}(d t, d h)\left(e^{-\lambda t}-e^{-\lambda t-\beta h}\right), \quad \beta, \lambda \geq 0,
$$

where $\mathrm{d}_{1} \geq 0$ and $\mu_{-}$is the Lévy measure of the bivariate descending ladder subordinator. It follows that for $q \geq 0$ fixed $\widehat{\kappa}(q, \cdot)$ is a Bernstein function. Since $\widehat{\kappa}(0, \beta)=\phi(\beta)$ for $\beta \geq 0$ and the formula in the last display holds for every $\lambda \geq 0$, we get that $\mathrm{d}_{1}=\mathrm{d}$. That is, the drift term of the Bernstein function $\widehat{\kappa}(q, \cdot)$ is equal to d. Moreover, it has been proved in Corollary 6 in [10] that the measure $\mu_{-}$can be written as

$$
\mu_{-}(d t, d h)=\int_{[0, \infty)} \mathcal{U}_{+}(d t, d s) \Pi(d h+s), \quad t, h>0,
$$


where $\mathcal{U}_{+}$denotes the potential measure of the ascending ladder subordinator. In our case $X$ is spectrally negative and hence due to the absence of positive jumps this formula becomes

$$
\mu_{-}(d t, d h)=\int_{[0, \infty)} \mathcal{U}_{+}(d t, d s) \Pi(d h+s)=\int_{[0, \infty)} d s \mathbb{P}\left(L_{s}^{-1} \in d t\right) \Pi(d h+s),
$$

see e.g. Exercise 7.5 in [20]. This allows us to write

$$
\begin{aligned}
\int_{(0, \infty)^{2}} \mu_{-}(d t, d h)\left(e^{-q t}-e^{-q t-\beta h}\right) & =\iiint_{(0, \infty)^{3}} d s \mathbb{P}\left(L_{s}^{-1} \in d t\right) \Pi(d h+s)\left(e^{-q t}-e^{-q t-\beta h}\right) \\
& =\iint_{(0, \infty) \times(0, \infty)} d s \Pi(d h+s)\left(e^{-s \Phi(q)}-e^{-s \Phi(q)-\beta h}\right) \\
& =\int_{0}^{\infty}\left(1-e^{-\beta h}\right) \int_{0}^{\infty} e^{-s \Phi(q)} \Pi(d h+s) d s, \quad \beta \geq 0 .
\end{aligned}
$$

As a consequence, for $q \geq 0$ fixed, the tail of the Lévy measure of $\widehat{\kappa}(q, \cdot)$ is given by

$$
\Upsilon_{q}(z, \infty):=\int_{z}^{\infty} \int_{0}^{\infty} e^{-s \Phi(q)} \Pi(d h+s) d s=e^{\Phi(q) z} \int_{z}^{\infty} d u e^{-\Phi(q) u} \Pi(u, \infty), \quad z>0 .
$$

This proves the claim about the tail of the Lévy measure. Using it we get that the Lévy measure of $\widehat{\kappa}(q, \cdot)$ has a density given by

$$
v_{q}(x):=\bar{\Pi}(x)-\Phi(q) e^{\Phi(q) x} \int_{x}^{\infty} e^{-\Phi(q) y} \bar{\Pi}(y) \mathrm{d} y, \quad x>0 .
$$

To prove that $v_{q}$ is non-increasing we observe first that an integration by parts leads to the equality

$$
v_{q}(x)=\bar{\Pi}(x)-\left(\bar{\Pi}(x)-e^{\Phi(q) x} \int_{x}^{\infty} e^{-\Phi(q) z} \pi(z) d z\right)=e^{\Phi(q) x} \int_{x}^{\infty} e^{-\Phi(q) z} \pi(z) d z,
$$

for $x>0$. Owing to the fact that $\pi$ is non-increasing, thanks to the assumption that it is a log convex Lévy density, we have that for $0<x<y$

$$
\begin{aligned}
v_{q}(x)-v_{q}(y) & =\Phi(q) e^{\Phi(q) x} \int_{x}^{y} e^{-\Phi(q) z} \pi(z) d z+\left(e^{\Phi(q) x}-e^{\Phi(q) y}\right) \Phi(q) \int_{y}^{\infty} e^{-\Phi(q) z} \pi(z) d z \\
& \geq \pi(y) e^{\Phi(q) x}\left(e^{-\Phi(q) x}-e^{-\Phi(q) y}\right)+\pi(y)\left(e^{\Phi(q) x}-e^{\Phi(q) y}\right) e^{-\Phi(q) y}=0,
\end{aligned}
$$

that is, $v_{q}$ is non-increasing.

Proof of Lemma 2.8. By assumption $\pi$ is a log convex Lévy density (and hence non-increasing) and then by the first paragraph of the proof of Theorem 2 in [14] we know that for $\beta \geq 0$, the functions $x \mapsto e^{-\beta x} \pi(x)$, and $\int_{x}^{\infty} e^{-\beta z} \pi(z) d z$, for $x>0$, are log convex. Hence, by taking $\beta=0$ we prove the claim about $\bar{\Pi}$ in Theorem 2.6. Furthermore, it then follows that the function $x \mapsto e^{\beta x} \int_{x}^{\infty} e^{-\beta z} \pi(z) d z$, for $x>0$, is $\log$ convex. We deduce the result claimed in Lemma 2.8 by taking $\beta=\Phi(q)$ and using the characterization of the Lévy density of $\widehat{\kappa}(q, \cdot)$ obtained in (4.6). 


\section{Acknowledgments}

The authors gratefully acknowledge funding from EPSRC grant numbers EP/E047025/1 and EP/C500229/1 and Royal Society grant number RE-MA1004. We are also grateful to Ronnie Loeffen for his comments on earlier drafts of this paper, in particular with regard to the computations around (4.4).

\section{References}

[1] Avram, F., Kyprianou, A.E. and Pistorius, M. R. (2004) Exit problems for spectrally negative Lévy processes and applications to (Canadized) Russian options. Ann. Appl. Probab. 14, 215238.

[2] Avram, F., Palmowski, Z. and Pistorius, M. R. (2007) On the optimal dividend problem for a spectrally negative Lévy process. Ann. Appl.Probab. 17, 156-180.

[3] Azcue, P. and Muler, N. (2005). Optimal reinsurance and dividend distribution policies in the Cramér-Lundberg model, Math. Financ. 15, 261-308.

[4] Bertoin, J. (1996) Lévy processes. Cambridge University Press, Cambridge.

[5] Bertoin, J. (1997) Exponential decay and ergodicity of completely asymmetric Lévy processes in a finite interval, Ann. Appl. Probab. 7, 156-169.

[6] Chan, T. and Kyprianou A.E. (2007) Smoothness of scale functions for spectrally negative Lévy processes. Preprint.

[7] De Finetti, B. (1957) Su un'impostazione alternativa dell teoria collecttiva del rischio. Transactions of the XVth International Congress of Actuaries 2, 433-443.

[8] Doney, R. A. (2005) Some excursion calculations for spectrally one-sided Lévy processes. Séminaire de Probabilités XXXVIII, 5-15, Lecture Notes in Math., 1857, Springer, Berlin.

[9] Doney, R. A. (2007) Fluctuation theory for Lévy processes. Lectures from the 35th Summer School on Probability Theory held in Saint-Flour, July 6-23, 2005. Lecture Notes in Mathematics, 1897.

[10] Doney, R. A.; Kyprianou, A. E. (2006) Overshoots and undershoots of Lévy processes. Ann. Appl. Probab. 16, 91-106.

[11] Fitzsimmons, P. J.; Fristedt, Bert; Shepp, L. A. (1985) The set of real numbers left uncovered by random covering intervals. Z. Wahrsch. Verw. Gebiete 70, 175-189.

[12] Furrer, H. (1998). Risk processes perturbed by $\alpha$-stable Lévy motion. Scand. Actuar. J. 59-74 
[13] Gerber, H.U. (1969) Entscheidungskriterien für den zusammengesetzten Poisson-Prozess. Schweiz. Verein. Versicherungsmath. Mitt. 69, 185-228.

[14] Gripenberg, G. (1978) On positive, nonincreasing resolvents of Volterra equations, J. Differential Equations, 30, 380-390.

[15] Gripenberg, G. (1980) On Volterra equations of the first kind, Integral Equations Operator Theory, 3, 473-488.

[16] He, S.W., Wang, J.G. and Yan, J.A. (1992) Semimartingale theory and stochastic calculus. Science Press, Beijing, New York.

[17] Hubalek, F. and Kyprianou, A.E. (2007) Old and new examples of scale functions for spectrally negative Lévy processes. arXiv:0801.0393v1 [math.PR].

[18] Huzak, M., Perman, M., Šikić, H. and Vondraček, Z. (2004a) Ruin probabilities and decompositions for general perturbed risk processes. Ann. Appl. Probab. 14, 1378-1397.

[19] Huzak, M., Perman, M., Šikić, H. and Vondraček, Z. (2004b) Ruin probabilities for competing claim processes. J. Appl. Probab. 41, 679-690.

[20] Kyprianou, A.E. (2006) Introductory Lectures on Fluctuations of Lévy Processes with Applications, Springer, Berlin.

[21] Kyprianou, A.E. and Rivero. V. (2008) Special, conjugate and complete scale functions for spectrally negative Lévy processes. To appear in Elec. J. Probab.

[22] Klüppelberg, C. and Kyprianou, A. E. (2006) On extreme ruinous behaviour of Lévy insurance risk processes. J. Appl. Probab. 43, 594-598.

[23] Klüppelberg, C., Kyprianou, A. E. and Maller, R. A. (2004) Ruin probabilities and overshoots for general Lévy insurance risk processes. Ann. Appl. Probab. 14, 1766-1801.

[24] Kyprianou, A.E. and Palmowski, Z. (2007) Distributional study of de Finetti's dividend problem for a general Lévy insurance risk process. J. Appl. Probab. 44, 349-365.

[25] Kyprianou, A. and Rivero, V. (2007) Special, conjugate and complete scale functions for spectrally negative Lévy processes. arXiv:0712.3588v1 [math.PR] To appear in Elec. J. Probab.

[26] Loeffen, R. L. (2007) On optimality of the barrier strategy in de Finetti's dividend problem for spectrally negative Lévy processes. To appear in Ann. Appl. Probab.

[27] Protter, P. (2005) Stochastic integration and differential equations. 2nd Edition, Springer, Berlin.

[28] Renaud, J-F. and Zhou, X. (2007) Distribution of the dividend payments in a general Lévy risk model. J. Appl. Probab. 44, 420-427.

[29] Rogers, L. C. G. (1984) A new identity for real Lévy processes. Ann. Inst. H. Poincaré Probab. Statist. 20, 21-34. 
[30] Song, R. and Vondraček, Z. (2006) Potential theory of special subordinators and subordinate killed stable processes, J. Theor. Prob. 19, 817-847.

[31] Song, R. and Vondraček, Z. (2007) On suprema of Lévy processes and application in risk theory. To appear in Annales de l'lnstitut Henri Poincaré.

[32] Song, R. and Vondraček, Z. (2007) Some remarks on special subordinators, To appear in Rocky Mount. J. Math.

[33] Winkel, M. (2002) Right inverses of non-symmetric Lévy processes. Ann. Probab. 30, 382-415. 